\title{
KANT E IL DIBATTITO SULLE QUESTIONI DI FINE VITA
}

\author{
KANT AND THE DEBATE ON END-OF-LIFE QUESTIONS
}

\author{
FEDERICA TRENTANI \\ $\left(\right.$ UFSC/Brasil $^{1}$
}

\begin{abstract}
In this article I claim that in a Kantian perspective the rational capacity of human beings can be considered as the core of moral life and, more generally, of human experience. On this basis I try to answer the following question: how should we conceive the value of life when the specifically human features are missing? More precisely, I suggest an antinaturalistic interpretation of Kantian theory concerning duties to oneself: my aim is to show that the ends of nature cannot work as a criterion for judging the correct use of our own dispositions. This reading can be applied to Kant's reflections on suicide, stressing that the Kantian view is a quite complex one and requires therefore careful consideration. Indeed, Kant does not conceive life a merely biological concept; this is the reason why I would like to point out that the Kantian perspective on these topics can provide an interesting conceptual background for the contemporary debate on end-of-life questions.
\end{abstract}

Keywords: Kant; antinaturalism; natural teleology; suicide; end-of-life.

\section{RIASSUNTO}

In questo articolo verrà sostenuto che in una prospettiva kantiana la capacità razionale dell'essere umano può essere considerata come il punto-chiave della vita morale e, più in generale, dell'esperienza umana. Su questa base si tenterà di rispondere alla seguente domanda: quale valore attribuire alla vita quando vengono a mancare proprio quelle caratteristiche che potremmo definire come specificamente umane? Più precisamente, verrà suggerita un'interpretazione antinaturalistica della teoria kantiana dei doveri verso se stessi: il mio obiettivo è infatti quello di mostrare che i fini della natura non possono funzionare come criterio per giudicare l'uso moralmente corretto delle nostre disposizioni. Questa lettura può inoltre essere applicata alle riflessioni di Kant sul suicidio, sottolineando così che la posizione kantiana è particolarmente complessa e richiede quindi un'attenta analisi. Kant non concepisce infatti la vita come un concetto meramente biologico; questo è il motivo per cui vorrei mettere in luce che la prospettiva kantiana può fornire un interessante background concettuale per il dibattito contemporaneo sulle questioni di fine vita.

Parole-chiave: Kant; antinaturalismo; teleologia naturale; suicidio; fine vita.

Note introduttive

In questo articolo il concetto kantiano di umanità verrà definito come la combinazione di razionalità, socievolezza e Kultur, ovvero come una nozione complessa e stratificata, sottolineando così il fatto che la nostra capacità razionale costituisce la condizione di possibilità dell'esperienza morale e, più in generale, dell'esperienza specificamente umana. Seguendo il filo rosso di queste riflessioni, si cercherà di rispondere alla seguente domanda: quale valore si può attribuire alla vita quando vengono meno le caratteristiche che rendono 'umano' un soggetto? Qui il termine 'umano' va pensato ethic@- Florianópolis v.12, n.2, p.227-237, Dez. 2013. 
prendendo le mosse da una definizione del concetto di umanità che si struttura in una molteplicità di piani e la cui componente più elementare è rappresentata dalla nostra capacità razionale.

Più precisamente, si tenterà di argomentare in favore di un'interpretazione antinaturalistica della teoria kantiana dei doveri verso se stessi con l'obiettivo di mostrare che i fini della natura non costituiscono il criterio-base per giudicare l'uso moralmente corretto delle nostre disposizioni. Questa lettura sarà poi messa a confronto con le pagine kantiane dedicate al tema del suicidio; in particolare, verrà sottolineato che la posizione di Kant su questo tema è più complessa di quanto ci si potrebbe aspettare: il discorso kantiano non si basa infatti su una concezione meramente biologica della vita. Per chiarire questo punto, proverò a delineare un esempio che permetta di individuare le condizioni che - in una prospettiva kantiana - rendono 'umana' una vita; nella parte finale dell'articolo verrà quindi evidenziato che le riflessioni di Kant su questi temi forniscono un background concettuale in grado di entrare in dialogo con il dibattito contemporaneo sulle questioni di fine vita.

\section{Antinaturalismo metaetico e teleologia naturale}

Per inquadrare la struttura metaetica della filosofia pratica kantiana, ${ }^{2}$ si può dire che alla sua base vi è la negazione del carattere prescrittivo del concetto di natura, ovvero il rifiuto di ogni forma di naturalismo metaetico; ${ }^{3}$ la teoria morale di Kant può quindi essere definita come una prospettiva metaetica oggettivistica e cognitivistica che fonda l'autonomia dell'etica nella distinzione tra fatti e valori: il tema portante che nelle Grundlegungsschriften permette a Kant di delineare il carattere normativo dell'etica e la sua autonomia dal mondo naturale è infatti la distinzione tra sein e sollen. Nella Metafisica dei costumi Kant si richiama all'impostazione antinaturalistica presentata nella Fondazione e nella seconda Critica sostenendo che l'antropologia costituisce soltanto il campo applicativo della morale (e non il suo fondamento di determinazione, il quale va invece ricondotto alla ragione pura pratica); Kant intende così mettere in luce non solo il fatto che l'etica non è fondata sulla natura, ma anche che l'obiettivo della sua filosofia pratica non è quello di scrivere un trattato sulla natura umana come aveva fatto, ad esempio, Hume:

noi dovremo prendere spesso ad oggetto la natura particolare dell'uomo, che è conosciuta soltanto attraverso l'esperienza, per mostrare in essa le conseguenze derivate dai principi morali universali, senza tuttavia offuscare la purezza di questi ultimi, né mettere in dubbio la loro origine a priori. Tutto questo per dire che una metafisica dei costumi non può certo essere fondata sull'antropologia, ma può esservi applicata. ${ }^{4}$ 
Nella Tugendlehre i doveri perfetti che il soggetto ha verso se stesso in quanto essere animale e morale vengono ricondotti al principio che prescrive di vivere secondo natura ${ }^{5}$ di fronte a questa affermazione è opportuno chiedersi se il vivere secondo natura sia un principio compatibile con l'etica kantiana nel suo insieme: in altre parole, Kant ha sempre sostenuto che la natura non è in grado di fornire principi morali che orientino la condotta degli esseri umani ed è proprio in base a questo convincimento che egli ha dato forma all'opposizione tra ragione e natura.

Va notato che la locuzione 'contrario alla natura dell'uomo' non ha necessariamente lo stesso significato di 'contrario alla natura intesa in senso generale'; più precisamente, si può dire che nella prospettiva di Kant è in gioco il dovere di preservare l'equilibrio fra le tre disposizioni della natura umana (l'animalità, l'umanità e la personalità): ${ }^{6}$ i doveri perfetti che il soggetto ha verso se stesso in quanto essere animale e morale vietano pertanto di trattare la propria natura animale in modi che ostacolino il mantenimento del proprio status di agente morale, ossia in modi che indeboliscano la capacità di utilizzare al meglio la propria facoltà razionale.

Nell'analizzare questo tipo di doveri, Kant fa riferimento anche alla teleologia naturale affermando che «[i doveri restrittivi o negativi] vietano all'uomo di agire contro il fine della propria natura e dunque [riguardano] soltanto l'autoconservazione morale». ${ }^{7}$ In relazione a questo punto è opportuno interrogarsi riguardo al significato morale dei fini della natura considerando due diverse interpretazioni; in base alla prima, nella quale la teleologia naturale viene intesa in senso forte, $i$ fini della natura fornirebbero indicazioni conclusive e moralmente vincolanti per l'uso delle nostre disposizioni: in questo caso il criterio della naturalità coprirebbe quindi lo stesso ambito applicativo del criterio della giustezza morale. ${ }^{8}$ In base alla seconda interpretazione, la quale attribuisce invece alla teleologia naturale un ruolo debole, i fini della natura riguardo al soggetto umano potrebbero essere considerati moralmente rilevanti, ma non decisivi: in altre parole, i fini della natura costituirebbero una sorta di sfondo-guida che orienta le nostre disposizioni animali, senza però determinare in modo preciso e vincolante quale sia il loro uso moralmente corretto.

Questa interpretazione 'debole' della teleologia naturale è focalizzata sul rapporto tra le disposizioni animali e la nostra natura razionale: il giudizio sull'uso moralmente corretto di queste disposizioni dipende dunque dal ruolo svolto dalla nostra natura animale in funzione di quella razionale. Dal momento che Kant pone a fondamento dei doveri verso se stessi il concetto di umanità, sembra più probabile ritenere che egli abbia assunto una posizione 'debole' riguardo al significato morale dei fini naturali; in questa prospettiva $i$ fini della natura riguardo alle nostre disposizioni animali hanno quindi un significato morale soltanto in relazione all'integrità e all'efficacia della nostra natura razionale. 
Sulla base di queste osservazioni è possibile sostenere - in primo luogo - che la concezione debole della teleologia naturale è la più appropriata da un punto di vista kantiano; in secondo luogo, che i fini della natura non costituiscono un argomento indipendente e conclusivo per il giudizio riguardo alla qualità morale delle azioni. ${ }^{9}$ Questo significa che al criterio della naturalità non deve essere attribuita una rilevanza morale maggiore di quella del concetto di umanità, il quale costituisce il fondamento dei doveri verso se stessi: il considerare come decisiva un'argomentazione basata soltanto sul criterio della naturalità significherebbe infatti accettare una forma di naturalismo metaetico, ovvero un'impostazione a cui Kant ha contrapposto le proprie riflessioni sulla morale. ${ }^{10}$

\section{Quando finisce la 'vita morale' del soggetto umano?}

Il concetto kantiano di umanità può essere inteso come la combinazione di due dimensioni che si completano reciprocamente: natura razionale e Kultur; la relazione che intercorre tra l'umanità e la cultura viene infatti evidenziata da Kant in diversi luoghi testuali, sia nella terza Critica che nella Metafisica dei costumi. ${ }^{11}$ Il concetto di umanità si riferisce quindi non solo alla capacità razionale di porsi fini e di selezionare i mezzi idonei al loro perseguimento, ma anche alla realizzazione progressiva di tutto ciò che caratterizza la sfera specificamente umana (compresa la Geselligkeit, ovvero la socievolezza); nel $\S 41$ della terza Critica Kant considera infatti l'impulso a entrare in società con altri esseri umani «come naturale per l'uomo, [...] e la socialità come una proprietà relativa ai bisogni dell'uomo in quanto creatura destinata alla società, dunque come una proprietà relativa all' umanità». ${ }^{12}$ Qui è importante sottolineare che alla base di questa complessa concezione della sfera umana vi è la nostra capacità razionale, la quale costituisce dunque la radice sulla quale si innestano tutte le ulteriori caratteristiche che definiscono il concetto di umanità.

A partire da queste premesse è interessante rileggere le pagine kantiane dedicate al tema del suicidio, quest'ultimo inteso come un atto che va considerato vizioso soltanto quando il soggetto tratta se stesso senza alcun riguardo per la propria natura razionale, ovvero senza alcun rispetto per l'umanità nella propria persona; il caso del suicidio mostra chiaramente che il principio del vivere secondo natura e il concetto di umanità sono criteri morali distinti a cui Kant attribuisce una diversa priorità: la sopravvivenza del soggetto (intesa come un fine naturale) viene infatti posta in secondo piano rispetto alla sua dignità morale in quanto essere dotato di ragione.

Per mettere a fuoco la questione, bisogna analizzare dettagliatamente le argomentazioni di Kant in proposito; una volta chiarito che il soggetto ha il dovere perfetto di non uccidersi, la posizione kantiana su questo tema è però più complessa di quanto ci si potrebbe aspettare: a questo riguardo va infatti evidenziato che Kant non basa il proprio discorso su una concezione meramente 
biologica della vita. ${ }^{13}$ Prima di argomentare in favore di questa tesi, è necessario commentare i passi della Tugendlehre in cui viene delineato il carattere vizioso del suicidio:

il primo, benché non il più importante, dovere dell'uomo verso se stesso in relazione alla propria animalità è l'autoconservazione della sua natura animale. Il contrario di questo dovere è la morte fisica volontaria. ${ }^{14}$ Annientare il soggetto della morale nella propria persona è come estirpare dal mondo [...] l'esistenza stessa della morale, che pure è un fine in sé. Dunque, disporre di se stessi come semplice mezzo in vista di un fine qualsiasi significa disprezzare l'umanità nella propria persona (homo noumenon), alla quale tuttavia l'uomo (homo phaenomenon) era affidato per la propria conservazione. ${ }^{15}$

In questa citazione i criteri per giudicare se si tratti di un atto vizioso di suicidio vengono individuati nella razionalità e nella 'capacità morale' dell'essere umano, il che significa che questi due criteri hanno la priorità rispetto alla vita intesa in senso meramente biologico: Kant sostiene infatti che è in relazione alla capacità di vivere l'esperienza morale che ciascuno ha il dovere di preservare il proprio status di possibile 'portatore' della volontà buona. ${ }^{16}$ La casistica morale della Tugendlehre menziona inoltre alcune circostanze in cui non è così facile stabilire se un'azione costituisca un atto vizioso di suicidio oppure no; ${ }^{17}$ senza prendere posizione in modo esplicito, Kant discute alcuni casi in cui il soggetto sembra essere moralmente autorizzato a togliersi la vita, ${ }^{18}$ motivo per cui sembra possibile considerare situazioni in cui il suicidio è permesso e non costituisce un'azione viziosa:

votarsi a morte certa (come Curzio) per salvare la patria è suicidio? Oppure il martirio volontario, il sacrificarsi per la salvezza della specie umana in generale, va considerato anch'esso un atto eroico? É lecito prevenire con il suicidio l'ingiusta condanna a morte da parte dell'autorità superiore, anche nel caso in cui quest'autorità permetta di farlo (come Nerone con Seneca)? [...] Un uomo sente già l'idrofobia come effetto del morso di un cane rabbioso e, [sapendo che non c'è possibilità di guarigione], si uccide per non rendere infelici altri uomini con la sua rabbia (di cui già sentiva i sintomi). Ci si chiede se egli abbia agito ingiustamente. $^{19}$

Anche se il testo non risponde a queste domande, si potrebbe interpretare l'incertezza di Kant come l'indicazione di alcuni casi concreti in cui il divieto di suicidarsi resta in secondo piano, lasciando così spazio ad altri aspetti moralmente rilevanti del contesto d'azione; il punto su cui concentrare l'attenzione risiede dunque nel fatto che in determinate situazioni il togliersi la vita non costituisce una mancanza di rispetto verso l'umanità nella propria persona. Nelle lezioni della Moralphilosophie Collins le circostanze che tolgono valore alla vita vengono definite come le ethic@-Florianópolis v.12,n.2,p.227-237, Dez. 2013. 
situazioni che privano l'essere umano della capacità di agire seguendo i principi della moralità 0 , più in generale, seguendo principi razionali. Per sottrarsi a questo tipo di circostanze, il soggetto sembra quindi essere autorizzato a togliersi la vita; Kant ritiene inoltre che questa azione estrema potrebbe essere determinata da un motivo buono, lo stesso motivo posto alla base delle azioni virtuose:

il suicidio possiede un aspetto apparentemente plausibile, allorché il prolungamento della vita dipenda da circostanze che possono annullare il valore della vita, quando cioè non si possa più vivere conformemente alla virtù e alla prudenza e quindi si debba porre fine alla vita per un nobile motivo. Coloro che difendono il suicidio sotto questo profilo adducono l'esempio di Catone [...]. Cosa doveva fare Catone? Sembra dunque che egli considerò necessaria la sua morte; egli pensò: dal momento che tu non puoi più vivere da Catone, allora non puoi più vivere affatto. Di fronte a questo esempio, si deve certo ammettere che, in un caso del genere, nel quale il suicidio è una virtù, le apparenze depongono fortemente in suo favore. Questo è l'unico esempio che dette al mondo l'occasione di difendere il suicidio. ${ }^{20}$

In relazione al caso di Catone va notato che un argomento-chiave è costituito dall'impossibilità di vivere la propria identità, ovvero dal non poter affermare la propria identità intesa come l'insieme dei principi in base a cui il soggetto orienta la propria vita e nei quali è possibile riconoscere se stessi in quanto esseri razionali capaci di moralità; Kant considera dunque alcuni casi nei quali il togliersi la vita può essere pensato come un sacrificio compiuto per preservare la propria moralità:

\begin{abstract}
dal momento che la vita va sacrificata in molte condizioni (quando io non possa preservare la vita se non attraverso la violazione dei doveri verso me stesso, allora sono vincolato a sacrificarla piuttosto che dover violare i doveri verso me stesso), così d'altra parte il suicidio non è permesso in alcuna condizione. [...] Vi sono cose nel mondo di gran lunga superiori alla vita. Osservare la moralità è di gran lunga più importante. È meglio sacrificare la vita piuttosto che perdere la moralità. ${ }^{21}$
\end{abstract}

Il passo appena citato permette di mostrare che, nel discutere del suicidio, Kant fa sempre riferimento a un soggetto dotato di capacità razionale, motivo per cui è opportuno interrogarsi sulla possibilità di rileggere le argomentazioni kantiane in una prospettiva più ampia, ovvero considerando il caso di un soggetto che si trovi a perdere progressivamente la propria capacità razionale. Le analisi di Kant potrebbero così costituire un punto di riferimento per il dibattito contemporaneo sulle questioni di fine vita; per chiarire l'ambito applicativo in cui sviluppare queste riflessioni verrà ora analizzato l'esempio di una persona che sta morendo lentamente a causa di una malattia degenerativa del sistema nervoso centrale. ${ }^{22}$ 
Nelle fasi iniziali di una patologia di questo tipo il soggetto potrebbe rendersi conto di non essere più in grado di contribuire alla felicità degli altri o allo sviluppo delle proprie disposizioni (non dimentichiamo che questi sono i due fini della ragione pura pratica che Kant pone al centro della propria teoria normativa) ${ }^{23}$ questa persona potrebbe inoltre essere consapevole del fatto che la propria capacità di fare esperienza di sé e del mondo verrà progressivamente cancellata. In un caso come questo il togliersi la vita non entrerebbe dunque in conflitto con i fini della ragione pura pratica; la domanda su cui riflettere può essere formulata in questi termini: quale valore si può attribuire alla vita quando vengono meno le caratteristiche che rendono 'umano' un soggetto? Chi sa di essere condannato a veder progressivamente cancellata la propria identità potrebbe anche dire: «No, questa 'non-vita' non la voglio; preferisco finire prima i miei giorni, piuttosto che sopravvivere senza essere me stesso».

Di fronte alla situazione appena delineata ci si può chiedere se abbia senso preservare (contro la volontà dell'interessato) la vita di un soggetto privo della capacità di essere moralmente attivo, di conoscere, sentire (fühlen) e pensare se stesso e ciò che lo circonda; in altre parole, ci si può chiedere se questa persona sia ancora un possibile 'portatore' della volontà buona. Nell'esempio qui analizzato l'umanità del soggetto sarebbe cancellata dal venir meno della capacità razionale intesa come la condizione di possibilità dell'esperienza morale e, più in generale, dell'esperienza specificamente umana; a sostegno di questa tesi si possono citare le parole con cui Kant descrive le circostanze che pongono fine alla vita morale del soggetto, mostrando così un'ulteriore implicazione del nesso fondamentale che lega i concetti di dignità umana, identità morale e autodeterminazione razionale: ${ }^{24}$

se non può preservare altrimenti la propria vita che disonorando la propria umanità, l'uomo deve piuttosto sacrificarla; senza dubbio, egli mette in pericolo la propria vita animale e tuttavia sente che, finché ha vissuto, ha vissuto degnamente. Non si tratta del fatto che l'uomo viva a lungo, ma del fatto che, finché vive, viva onorevolmente e che non disonori la dignità dell'umanità; se non può più vivere così, egli non può più vivere affatto e la sua vita morale è giunta al termine. ${ }^{25}$ 


\begin{abstract}
${ }^{1}$ Bolsista Pós-Doutorado Júnior do CNPq na Universidade Federal de Santa Catarina - Departamento de Filosofia.
${ }^{2}$ Riguardo all'impostazione metaetica della morale kantiana cfr. S. LANDUCCI, Sull'etica di Kant, Milano, Guerini e Associati, 1994, pp. 15-67.

${ }^{3}$ Alcuni interpreti hanno invece sostenuto che la filosofia pratica kantiana potrebbe essere intesa come una teoria naturalistica; secondo Moore con la teoria del fatto della ragione anche Kant cadrebbe nella cosiddetta fallacia naturalistica: cfr. G. E. MoORE, Principia Ethica, Cambridge, Cambridge University Press, 1903, §§ 76-77. Ilting ritiene che sia la Fondazione l'opera in cui Kant cadrebbe nella fallacia naturalistica: cfr. K. H. ILTING, Der naturalistische Fehlschluß bei Kant, in ID., Grundfragen der praktischen Philosophie, Frankfurt a.M., Suhrkamp, 1994, pp. 277-295. Per una critica alle argomentazioni di Moore sulla fallacia naturalistica in Kant cfr. S. LANDUCCI, Sull'etica di Kant, cit., pp. 20, 60-62.
\end{abstract}

${ }^{4}$ MS, AA VI 217 / 33. Le opere di Kant vengono citate facendo riferimento alla Akademie-Ausgabe e alle traduzioni italiane qui indicate: I. KANT, Metafisica dei costumi, trad. it. di G. Landolfi Petrone, Milano, Bompiani, 2006; I. KANT, Lezioni di etica, trad it. di A. Guerra, Roma-Bari, Laterza, 1984; I. KANT, Fondazione della metafisica dei costumi, trad. it. di P. Chiodi, in ID., Scritti morali, Torino, UTET, 1995; I. KANT, Critica della capacità di giudizio, trad. it. di L. Amoroso, Milano, Rizzoli, 1995.

5 «Il primo principio del dovere verso se stessi risiede nel detto: vivi secondo natura (naturae convenienter vive), ovvero conservati nella perfezione della tua natura» (MS, AA VI 419 / 453). Riguardo alla nozione di dovere perfetto è utile ricordare che nella Tugendlehre Kant suddivide i doveri di virtù in base alla maggiore o minore determinatezza delle massime da adottare, sottolineando così che l'applicazione della legge morale ai casi concreti può essere portata avanti in modi molto diversi, a seconda del tipo di dovere preso in esame. Kant riflette inoltre sul 'modo' in cui un dovere obbliga il soggetto, considerando anche la distinzione tra obbligazione larga e obbligazione stretta, quest'ultima riferita ai doveri perfetti che prescrivono di compiere (o di astenersi dal compiere) azioni specifiche; i doveri imperfetti sono invece di obbligazione larga e prescrivono massime volte a promuovere i fini della ragione pura pratica.

${ }^{6}$ Per questa osservazione cfr. L. DENIS, Moral Self-Regard. Duties to Oneself in Kant's Moral Theory, New York, Garland, 2001, p. 98.

${ }^{7} M S$, AA VI $419 / 451$.

${ }^{8}$ Questa distinzione tra i due modi di intendere la teleologia naturale in riferimento alla sfera umana viene proposta da L. DENIS, Moral Self-Regard, cit., p. 107.

${ }^{9}$ Riguardo a queste osservazioni sulla questione della teleologia naturale cfr. P. GUYER, Ends of reason and ends of nature: the place of teleology in Kant's ethics, in ID., Kant's system of nature and freedom. Selected essays, Oxford, Clarendon Press, 2005, pp. 172, 193-197.

${ }^{10}$ Aportone fa notare che le argomentazioni kantiane sul suicidio vanno inquadrate entro una prospettiva antinaturalistica: cfr. A. APORTONE, La morte volontaria negli scritti di Kant e nelle riflessioni di un anonimo, in ID. (a cura di), Sull'etica del suicidio. Dalle riflessioni e lezioni di Immanuel Kant con i Preparativi di un infelice alla morte volontaria di un Anonimo del Settecento, Firenze, Le Lettere, 2003, p. 34.

${ }^{11}$ Cfr. KU, AA V $431 / 745 ; M S$, AA VI 392 / 399; MS, AA VI 444 / 507.

${ }^{12} K U$, AA V 296-297 / 399.

13 «La vita non va in nessun modo stimata in sé e per sé, ma al contrario io devo cercare di conservare la mia vita solo nella misura in cui sono degno di vivere» (MPCollins, AA XXVII 371 / 172). Cfr. anche: «per quel che concerne il dovere riguardo all'aver cura della nostra vita è da notare che la vita in sé e per sé non è il bene maggiore che ci sia stato affidato e del quale dobbiamo aver cura. Ci sono doveri ben più grandi della vita e che spesso devono essere adempiuti con il sacrificio della vita» (MPCollins, AA XXVII 375 / 177); «vi sono doveri a cui la vita è subordinata e per l'adempimento dei quali non dobbiamo lasciar trasparire alcuna viltà riguardo alla nostra vita» (MPCollins, AA XXVII 376 / 178).

${ }^{14} M S$, AA VI $421 / 457$. 


\begin{abstract}
${ }^{15} M S$, AA VI $423 / 461$.
${ }^{16}$ Riguardo alla 'potenzialità morale' del soggetto kantiano cfr. F. RICKEN, Homo noumenon und homo phaenomenon. Ableitung, Begründung und Anwendbarkeit der Formel von der Menschheit als Zweck an sich selbst, in O. Höffe (Hrsg.), Grundlegung zur Metaphysik der Sitten. Ein kooperativer Kommentar, Frankfurt/Main, 2000.
\end{abstract}

${ }^{17}$ Gregor sottolinea che le questioni casistiche legate al suicidio vanno ricondotte al problema del conflitto di doveri: cfr. M. J. GREGOR, Laws of freedom. A study of Kant's method of applying the categorical imperative in the Metaphysik der Sitten, Oxford, Blackwell, 1963, p. 135. Rawls propone un'interpretazione simile a quella di Gregor: cfr. J. RAWLS, Lectures on the History of Moral Philosophy, Cambridge Mass., Harvard University Press, 2000, p. 193. Anche Aportone rilegge il tema del suicidio facendo riferimento al problema del conflitto tra doveri: cfr. A. APORTONE, La morte volontaria negli scritti di Kant e nelle riflessioni di un anonimo, cit., p. 47. Per un diverso punto di vista riguardo alle questioni casistiche legate al suicidio cfr. Y. UNNA, Kant's answers to the casuistical questions concerning self-disembodiment, «Kant-Studien», 94, 2003, pp. 458, 472-473. Cfr. anche S. B. KIM, The formation of Kant's casuistry and method problems of applied ethics, «Kant-Studien», 100, 2009, pp. 339-340.

${ }^{18}$ Secondo Wittwer la prospettiva delle questioni casistiche permette di tenere conto della differenza tra il togliersi la vita (Selbsttötung) e il suicidarsi (Selbstmord): cfr. H. WITTWER, Über Kants Verbot der Selbsttötung, «Kant-Studien», 92, 2001, p. 209. Cfr. D. N. JAMES, Suicide and stoic ethics in the Doctrine of Virtue, «Kant-Studien», 90, 1999 , p. 48.

${ }^{19} M S$, AA VI 423-424 / 461, 463.

${ }^{20}$ MPCollins, AA XXVII 370-371/171.

${ }^{21}$ MPCollins, AA XXVII 372-373 / 173-174.

${ }^{22}$ James suggerisce di considerare la questione casistica dell'uomo morso dal cane rabbioso come una situazione che per molti aspetti è simile a quella dei pazienti affetti dall'Alzheimer: cfr. D. N. JAMES, Suicide and Stoic Ethics in the Doctrine of Virtue, cit., p. 57. Aportone fa notare che le riflessioni di Kant sul tema del suicidio «portano verso la soglia della discussione contemporanea sull'eutanasia, che non riguarda tanto la massima del suicidio come principio di conduzione della vita che sottomette l'umanità alle condizioni sensibili, quanto la pretesa di derivare un diritto alla morte volontaria da una massima fondata sul rispetto dell'umanità nella persona condizionata dalla fragilità del suo stato sensibile. [...] In questo rispetto, Kant può offrirci dei principi di giudizio, ma non indicazioni precise, dato che il problema dell'eutanasia ha assunto oggi caratteri e dimensioni per lui inimmaginabili, per esempio la possibilità tecnica di mantenere le funzioni vitali del corpo in assenza di una vita personale. [...] [Queste riflessioni] lasciano di fatto aperta la possibilità che la condanna kantiana della massima del suicidio possa convivere con la giustificazione di regole pratiche e quindi di azioni che, sotto condizioni non specificabili a priori, abbreviano la vita» (A. APORTONE, La morte volontaria negli scritti di Kant e nelle riflessioni di un anonimo, cit., pp. 53-54).

${ }^{23}$ Nella Dottrina della virtù Kant delinea la sfera dell'etica attraverso due fini che sono allo stesso tempo doveri: il perfezionamento di sé e la felicità altrui. Per un'introduzione complessiva alla Metafisica dei costumi cfr. M. TIMMONS (ed.), Kant's Metaphysics of Morals. Interpretative Essays, Oxford, Oxford University Press, 2002; L. DENIS (ed.), Kant's Metaphysics of Morals. A Critical Guide, Cambridge, Cambridge University Press, 2010. Sulla Tugendlehre cfr. O. SEnsen - J. Timmermann - A. Trampota (ed.), Kant's Tugendlehre. A Comprehensive Commentary, Berlin-New York, de Gruyter, 2013.

${ }^{24}$ «L'autonomia è il principio della dignità della natura umana e di ogni natura razionale» (GMS, AA IV 436 / 95). Riguardo al concetto di dignità cfr. anche i seguenti passi: GMS, AA IV 434 / 94; GMS, AA IV 440 / 99; MS, AA VI 436 / 489; MS, AA VI 462 / 547.

${ }^{25}$ MPCollins, AA XXVII 377 / 179. 


\section{Bibliografia}

APORTONE, Anselmo. La morte volontaria negli scritti di Kant e nelle riflessioni di un anonimo. In: APORTONE, Anselmo (a cura di). Sull'etica del suicidio. Dalle riflessioni e lezioni di Immanuel Kant con i Preparativi di un infelice alla morte volontaria di un Anonimo del Settecento. Firenze: Le Lettere, 2003.

DENIS, Lara. Moral Self-Regard. Duties to Oneself in Kant's Moral Theory. New York: Garland, 2001.

(ed.). Kant's Metaphysics of Morals. A Critical Guide. Cambridge: Cambridge University Press, 2010.

GREGOR, Mary J. Laws of freedom. A study of Kant's method of applying the categorical imperative in the Metaphysik der Sitten. Oxford: Blackwell, 1963.

GUYER, Paul. Ends of reason and ends of nature: the place of teleology in Kant's ethics. In: GUYER, Paul (ed.). Kant's system of nature and freedom. Selected essays. Oxford: Clarendon Press, 2005.

ILTING, Karl H. Der naturalistische Fehlschluß bei Kant. In: ILTING, Karl H. (Hrsg.). Grundfragen der praktischen Philosophie. Frankfurt a.M.: Suhrkamp, 1994.

JAMES, David N. Suicide and stoic ethics in the Doctrine of Virtue. In: Kant-Studien, n. 90, 1999.

KANT, Immanuel. Gesammelte Schriften. Hrsg.: Bd. 1-22 Preußische Akademie der Wissenschaften, Bd. 23 Deutsche Akademie der Wissenschaften zu Berlin, ab Bd. 24 Akademie der Wissenschaften zu Göttingen. Berlin, $1900 \mathrm{ff}$.

_. Metafisica dei costumi. Trad. Giuseppe Landolfi Petrone. Milano: Bompiani, 2006.

_. Lezioni di etica. Trad. Augusto Guerra. Roma-Bari: Laterza, 1984.

Torino: UTET, 1995.

Fondazione della metafisica dei costumi. Trad. Pietro Chiodi. In: Scritti morali, . Critica della capacità di giudizio. Trad. Leonardo Amoroso. Milano: Rizzoli, 1995.

KIM, Soo B. The formation of Kant's casuistry and method problems of applied ethics. In: Kant-Studien, n. 100, 2009.

LANDUCCI, Sergio. Sull'etica di Kant. Milano: Guerini e Associati, 1994.

MOORE, George E. Principia Ethica. Cambridge: Cambridge University Press, 1903.

RAWLS, John. Lectures on the History of Moral Philosophy. Cambridge Mass.: Harvard University Press, 2000. 
RICKEN, Friedo. Homo noumenon und homo phaenomenon. Ableitung, Begründung und Anwendbarkeit der Formel von der Menschheit als Zweck an sich selbst. In: HÖFFE, Otfried (Hrsg.). Grundlegung zur Metaphysik der Sitten. Ein kooperativer Kommentar. Frankfurt a.M, 2000.

SENSEN, Oliver - TIMMERMANN Jens - TRAMPOTA Andreas (ed.). Kant's Tugendlehre. A Comprehensive Commentary, Berlin-New York: de Gruyter, 2013.

TIMMONS, Mark (ed.). Kant's Metaphysics of Morals. Interpretative Essays. Oxford: Oxford University Press, 2002.

UNNA, Yvonne. Kant's answers to the casuistical questions concerning self-disembodiment. In: Kant-Studien, n. 94, 2003.

WITTWER, Héctor. Über Kants Verbot der Selbsttötung. In: Kant-Studien, n. 92, 2001. 